\title{
Multicentre epidemiological survey on the incidence of Streptococcus agalactiae in pregnancy
}

\author{
Leonardo Lodolo', Michelangelo Barbaglia', Claudia Canale', Cinzia Rossi', Andrea Guala², Nino Cappuccia' \\ I SOC Laboratorio di Analisi Chimico-Cliniche e Microbiologiche, ASL-VCO, Verbania \\ 2 SOC Pediatria e Neonatologia , ASL-VCO, Verbania.
}

Key words: incidence of S. agalactiae (GBS) in pregnancy, GBS Study Group of Piemonte and Valle d'Aosta, vaginal and vagino/rectal swabs, Confidence Interval $(\mathrm{Cl})$ of positivity, enrichment in broth.

\section{Indagine epidemiologica multicentrica sull'incidenza di S. agalactiae in gravidanza}

\section{SUMMARY}

B-hemolytic streptococci group B (GBS) cause infections of the urinary tract, endometrium, amnion, and superinfection of wounds, with complications in $2 \%-4 \%$ of cases in pregnant woman. The infection transmitted to newborns is a cause of sepsis and meningitis with high incidence of mortality.A recent report on a multicentric study of the ISS, showed that detection of S. agalactiae in pregnant women in different realities of healthcare, ranging from $3.9 \%$ to $19.4 \%$. This survey covers a sample of 29607 women screened in a total of 36852 childbirths, with a positivity of 4183 subjects equal to $14.1 \%$.

Thanks to the GBS Study Group of Piemonte and Valle d'Aosta microbiology laboratories, with the support of 42 study centres have been involved. Collection techniques, types of medium, environmental of incubation of the culture, possible enrichment in broth, of the swabs of 2849 I women during $2006 / 2007$ have been considered. $24.36 \%$ were vaginal swab while $75.64 \%$ vagino/rectal.

According to data collected at 3 I centers with eligible data (22, I75 pregnant) GBS positivity was $12.7 \%$ (Cl [Confidence Interval] + / $-95 \%$ : $13.1 \%-12.2 \%)$ with wide variability between individual centers $(2 \%-22.6 \%)$. The enrichment broth is used by 6 out of 9 centers with positivity> $15 \%$ (M: 20.05\%), from 6 to 14 with positivity between $10-15 \%$ (M: $12.61 \%$ ), from none to 8 centers with less than $10 \%$ positivity (M: $4.95 \%)$. This multicentre survey underlined the preference for vagino/rectal swab, as recommended by the literature.

The frequency of GBS positivity of pregnant women on the whole samples is similar to that found in other national surveys, even if with large differences between the different centers. The sensitivity tends to increase with the use of enrichment techniques, whose use is uneven between the centers, perhaps indicating the need for greater uniformity of protocols based on the results of microbiological analysis EBM (Evidence Based Medicine).

\section{La ricerca dello stato di portatore di Streptococcus agalactiae}

Streptococcus agalactiae (SGB) nella gravida è lo standard di prevenzione delle infezioni neonatali precoci. Non mancano in letteratura innumerevoli riferimenti, su procedure standard e linee guida $(4,5,6,9)$, con indicazioni precise di come attuare screening nelle donne gravide a 35-37 settimane di gestazione (11). Nonostante tali indicazioni i dati riscontrabili in letteratura sulle positività di tali screening presentano una variabilità difficilmente attribuibile a fattori ambientali (12).

\section{Scopo dell'indagine}

Da un recente rapporto dell'Istituto Superiore di Sanità (ISS) (10) su uno studio multicentrico, la positività nelle gestanti varia dal $3.9 \%$ al $19.4 \%$. Con una media di $14.1 \%$ su un campione di 29607 donne sottoposte a screening.

Un'ampia bibliografia internazionale colloca tra il $10 \%$ ed il 30 \% (16) le donne in età fertile colonizzate a livello vaginale e/o rettale da SGB (2).

L'efficacia del metodo usato per la ricerca di SGB è soggetta a variabili legate all'accuratezza operativa comprendente:

- il periodo di esecuzione: la $35^{\mathrm{a}}-37^{\mathrm{a}}$ settimana di gestazione è il più indicato ad assicurare sensibilità e specificità (13);

- la fase preanalitica, caratterizzata dall'accuratezza del prelievo che può essere effettuato, mediante lo stesso tampone, prima a livello vaginale e poi rettale (1). Tali tamponi, immessi in terreni di trasporto, non nutritivi (di Amies, di Stuart), mantengono la vitalità degli SGB per almeno $48 \mathrm{~h}$ a temperatura ambiente o a $4-8{ }^{\circ} \mathrm{C}$;

- la fase analitica, la cui efficacia, condizionata in parte dai materiali usati, risulta dipendere in particolare dai protocolli seguiti. È consigliabile l'utilizzo di un brodo di arricchimento e terreno solido selettivi (3);

La fase postanalitica, che consiste nel sottoporre il ceppo isolato di SGB alle prove di sensibilità agli antibiotici, può limitarsi al test (D-test) per Eritromicina e Clindamicina, mediante il quale, oltre che a rilevare la resistenza verso i macrolidi, è possibile evidenziare l'inducibilità della resistenza anche nei confronti dei lincosamidi.

L'antibiogramma può essere utile, oltre che per verificare la possibilità di usare i macrolidi quale valida alternativa ai ß-lattamici nella paziente allergica, anche per evidenziare eventuali fenotipi di resistenza verso macrolidi-lincosamidi-streptogramine B (MLSB) e tetracicline. Verso queste ultime la resistenza di S. agalactiae è intorno all' $80-90 \%$.

Questo studio vuole analizzare i protocolli d'indagine seguiti nella Regione Piemonte e Valle d'Aosta.

Sono stati contattati 70 laboratori tramite una scheda per la raccolta dei dati alla ricerca dello SGB in gravidanza.

Dei laboratori contattati:

- 23/70 non hanno risposto

- 5/47 hanno fornito dati non interpretabili ai fini dell'indagine

- 4/47 hanno comunicato di non disporre di un settore di Microbiologia

- 3/47 hanno fornito tutti i dati tranne le percentuali di positività

- 4/47 hanno fornito solo il numero delle indagini effettuate senza rispondere alle altre domande

- 31/47 hanno fornito tutti i dati richiesti

Sono state analizzate:

- tecniche di raccolta e numero dei campioni/anno

- tipologia dei terreni di coltura, selettivi, cromogenici e brodi di arricchimento

- atmosfera d'incubazione delle colture

- sistemi di identificazione

- allestimento di prove di sensibilità agli antibiotici

- utilizzo di controlli di qualità interni ed esterni

- tipo di refertazione (qualitativa, semiquanitativa, quantitativa)

Lo studio è stato condotto su tamponi provenienti da 28491 donne nel 2006-2007: 24.36\% tamponi solo vaginali e 75.64\% vagino/rettali.

\section{Corresponding author: Leonardo Lodolo}

SOC Laboratorio Analisi - Sez. Microbiologia ASL-VCO - Ospedale Castelli

28887 Verbania - Tel.: 0323868929 - Fax: 0323868925

E-mail: labana.om@aslvco.it 
Su 31 Centri con dati eligibili (22175 gravide) la positività media per SGB è risultata del $12.7 \%$ con un CI (Confidence Interval) $+/-95 \%$ da $13.1 \%$ al $12.2 \%$, con ampia variabilità fra i singoli Centri (2\%-22\%).

I dati raccolti evidenziano una situazione abbastanza disomogenea:

- tecniche di raccolta e numero dei campioni/anno

- l'utilizzo, come terreno solido selettivo, di Columbia agar + $5 \%$ di sangue + CNA (Colistina, acido nalidixico), riguarda il 78\% dei laboratori, corrispondente all' $84 \%$ dei tamponi; il 13\% dei laboratori utilizza terreni non selettivi arricchiti con il 5\% di sangue di montone (corrispondente al 7\% del totale dei tamponi); solo il 9\% dei laboratori utilizza terreno cromogenico (corrispondente al 9\% del totale dei tamponi);

- il ricorso all'arricchimento in brodo riguarda solo il 35\% dei laboratori (che lavorano il 44\% dei tamponi totali)

- Il $67 \%$ utilizza $\mathrm{CO}_{2} 10 \%$, per l'incubazione in atmosfera controllata (sul 75\% dei tamponi totali); il 24\% dei laboratori (sul 19\% dei tamponi totali) utilizza l'incubazione in anaerobiosi; il 9\% dei laboratori utilizza l'incubazione in atmosfera ambiente (sul 6\% dei tamponi totali).

- nell’81\% dei laboratori si utilizza, quale metodo di identificazione, l'agglutinazione al lattice (sul 75\% dei tamponi totali), il $24 \%$ utilizza anche l'identificazione biochimica (18\% dei tamponi totali), il 18\% infine utilizza la sola identificazione biochimica (25\% dei tamponi totali).

Le percentuali di positività rilevate, messe a confronto con gli altri dati analizzati, hanno messo in evidenza una significativa variabilità dovuta all'utilizzo, o meno, dell'arricchimento in brodo.

I campioni che utilizzano l'arricchimento in brodo presentano una positività media del 15.49\% (CI + 95\%: 14.78\%-16.23\%). I campioni processati senza arricchimento in brodo presentano invece una positività media per SGB del 10.53\% (CI + 95\%: $10-11.08 \%$ ).La differenza tra i gruppi è altamente significativa $\left(\mathrm{p}<0.0000001\right.$ utilizzando il test del $\chi^{2}$ ).

Questo studio presenta un campione sufficientemente significativo ed evidenzia un'ampia difformità nei diversi metodi di indagine seguiti, quale la mancanza di una precisa standardizzazione delle metodiche e, principalmente, il non utilizzo dell'arricchimento in brodo, consigliato da numerose linee guida. La falsa negatività di un tampone vagino-rettale per streptococco potrebbe essere fuorviante nella gestione clinica del neonato. Essa peraltro può dipendere da vari fattori come la non corretta tecnica di prelievo, la impropria conservazione o trasporto del campione, la intermittente eliminazione del germe nelle portatrici, ecc... (8). In base ai nostri risultati si può ipotizzare che colture "false negative" lo siano soprattutto per il mancato rispetto delle linee guida microbiologiche in laboratorio ed in particolar modo per il mancato arricchimento in brodo.

Si rende quindi auspicabile una standardizzazione dei metodi d'indagine microbiologica nelle diverse realtà, anche per avere dati più confrontabili; le possibili differenze epidemiologiche possono infatti essere alterate da metodi d'indagine difformi. Nuovi scenari si aprono con la possibilità di utilizzare metodi rapidi d'indagine utilizzando tecniche di biologia molecolare (7), ma per il momento lo screening in gravidanza, mediante indagine colturale, dopo arricchimento in brodo, su tampone vagino/rettale, rappresenta il gold standard nella prevenzione delle infezioni da SGB nel neonato. Per le donne che si presentano al parto, senza essere state sottoposte a tale indagine, può essere utile disporre di test rapidi immunocromatografici da effettuarsi nel peripartum. Sarà comunque con l'introduzione della vaccinazione nella puerpera, per lo SGB, che si modificherà sostanzialmente il quadro epidemiologico dei portatori e l'approccio clinico alla puerpera ed al neonato.

\section{BIBLIOGRAFIA}

1. Badri MS, Zawaneh S, Cruz AC et al. Rectal colonization with group B streptococcus: relation to vaginal colonization of pregnant

2. Berardi A, Lugli L, Baronciani D, et al. Group B streptococcal infections in a northern region of Italy. Pediatrics 2007;120:e487-e93.

3. CDC. Laboratory practices for prenatal group B streptococcal screening and reporting. Connecticut, Georgia and Minnesota, 1997-1998. MMWR 1999;48:426-8.

4. CDC. Prevention of perinatal group B streptococcal disease. MMWR 2002;51:1-22

5. Comitato di Studio AMCLI per le Infezioni Sessualmente Trasmesse (CoSIST). Linee guida per le indagini diagnostiche microbiologiche nello studio delle infezioni uretro-cervico-vaginali (2001).

6. Consiglio Direttivo SIMP (Società Italiana Medicina Perinatale). Lineeguida per la prevenzione delle infezioni perinatali da streptococco di gruppo B. Boll Soc Ital Med Perinat 1996;1:21-4.

7. Edwards RK, Novak-Weekley SM, Koty PP, Davis T, Leeds LJ, Jordan JA Rapid group B streptococci screening using a real-time polymerase chain reaction assay. Obstet Gynecol. 2008 Jun;111(6):1335-41)

8. Hansen SM, Uldbjerg N, Killian M, Sorensen UB. Dynamics of Streptococcus Agalactiae colonization in women during and after pregnancy and in their infants. J Clin Microbiol 2004;42:83-9.

9. Health Protection Agency (2006). Processing swabs for Group B streptococcal carriage. National Standard Method BSOP 58 Emissione 2.

10. ISS. Infezioni da streptococco di gruppo B. Rapporto Istisan 07/28, Roma, 2007. 11. Persson K, Christensen KK, Christensen P, Forsgren A, Jorgensen C,
Persson PH, Asyntomatic bacteriuria durimg pregnancy with spezial reference to group B streptococci. Scand J Infect Dis 1985;17:195-9.

12. Trijbels-Smeulders MA, Kollee MA, Adriaanse AH, Kimpen JL, Gerards LJ Neonatal group B streptococcal infection: incidence and strategies for prevention in Europe. Pediatr Infect Dis J 2004;23:172-3.

13. Yancey MK, Schucat A, Brown LK, Ventura VL, Markenson GR. The accuracy of late antenatal screening cultures in predicting genital group B streptococcal colonization at delivery. Obstet Gynecol 1996;88:811-5. 